\title{
SmartNotes: Application of Crowdsourcing to the Detection of Web Threats
}

\author{
Mehrbod Sharifi \\ mehrbod@cs.cmu.edu
}

\author{
Eugene Fink \\ eugenefink@cmu.edu
}

\author{
Jaime G. Cabonell \\ jgc@cs.cmu.edu
}

Computer Science, Carnegie Mellon University, Pittsburgh, PA 15217

\begin{abstract}
We describe a crowdsourcing system, called SmartNotes, which detects security threats related to web browsing, such as Internet scams, deceptive sales of substandard products, and websites with intentionally misleading information. It combines automatically collected information related to the website reputation with user votes and comments, and uses it to identify potential threats. We have implemented it as a browser extension, which is available for free public use.
\end{abstract}

Keywords-Crowdsourcing, Machine Learning, Web Security.

\section{INTRODUCTION}

Cybersecurity threats can be broadly divided to two high-level types. The first type is the threats caused by factors outside the end user's control, such as security flaws in applications and protocols. Examples include drive-by downloads [Cova et al. 2010], where an attacker exploits the web browser's vulnerability to install malicious code; and cross-site request forgery [Barth et al. 2008], where an attacker uses a trusted browser session to steal or affect sensitive information. The traditional remedy is distributing software patches and amending protocols. Many security applications are also designed to protect the users from these threats.

The work on defenses against such security threats is still an ongoing battle, but there is another critical type of cybersecurity threats, which are caused by actions on the part of the user. Web browsers and operating systems have improved to include more precautions against security threats, but the attackers find ways to trick the users into bypassing the security barriers. For example, an attacker may convince victims that their computers are already infected and have them install a fake anti-virus. The number of such threats has been growing in recent years as more technically naïve people conduct their daily activities through the web, thus providing the attackers with opportunities to exploit the users' naïveté. For example, phishing [Kumaraguru at al., 2009] and other similar scams have emerged due to the increased popularity of on-line banking and social networking. While novice users are most vulnerable, evidence suggests that experts may also be affected in sophisticated cases.

The research in this area is in a very preliminary stage [Sharifi et al., 2010]. The detection of such threats is a difficult problem for machine learning algorithms, partly because there is no large datasets of malicious websites for most threats; however many on-line services offer partial relevant information for identifying some of the malicious websites. For example, the statistics available from alexa.com and similar web metrics companies can help spotting websites with high traffic, which are usually safe. The problem with this approach is that we cannot rely on any one statistic or source of information. For example, some high-traffic websites, such as wordpress.com, allow users to host scam contents in their subdomains. Another commonly used information source for identifying malicious websites is blacklists, which are websites maintaining a list of suspicious websites, usually based on some form of community reports (e.g., spamcop.net, hostsfile.net). The main limitations of the blacklist approach are well-known. Specifically, (1) a list may not include new scam websites (false negatives), (2) it can mistakenly include legitimate websites (false positives), and (3) scammers may intentionally bias blacklists in both directions.

To address these problems, we have taken a crowdsourcing approach. We encourage users to identify and report security threats, and then we apply machine learning to integrate their responses. This idea is analogous to user-review mechanisms, where people share their experiences with specific products or services. The unique characteristics of our developed system are as follows:

1) Applying machine learning and language processing to analyze and integrate user feedback. This part will be described in future papers.

2) Combining the information from multiple heterogeneous sources.

3) Combining social bookmarking with question-answering to encourage wider user participation.

From the users' point of view, the developed system acts as a personal security assistant, which automatically collects relevant information and helps the users identify websites that may pose a threat.

\section{RELATED WORK}

The described work is related to three main areas: social bookmarking, question answering, and safe browsing. We review each of these areas and then describe the related work on detection of web scam. 


\section{A. Social Bookmarking}

The social bookmarking is a mechanism for sharing bookmarks among users, often with categorization, comments, and other additional information. One of the most popular social bookmarking services is delicious.com. This idea has evolved during the past decade into more advanced services, such as website discovery (e.g., stumbleupon.com), news discovery (e.g., reddit.com and digg.com) and social network sharing (e.g., addthis.com).

We implemented SmartNotes as a specialized social bookmarking tool with focus on web security. The users not only bookmark interesting websites but also identify and rate potentially malicious websites, and provide comments on related threats.

\section{B. Question Answering}

Question-answering services enable users to post questions and answer question posed by others. The most popular services include Yahoo Answers (answers.yahoo.com), Google Answers (answers.google.com), and Quora (quora.com). Some websites are focused on questions in specific areas, such as for example computer programming (e.g., stackoverflow.com) or security (e.g., scam.com).

The question-answering system within SmartNotes is for asking questions about specific websites. We have designed it to encourage conversations among users, and to enable novice users to ask experts about potential threats. While experts are often unwilling to invest their time into identifying malicious websites, they may contribute if related inquiries come from their friends through an easy to use question-answering mechanism. In addition to obtaining expert feedback, we also gather data on the pattern of communications among users, which helps in detecting biases in user opinions.

\section{Safe Browsing}

Safe browsing tools assist users in detecting and blocking web security threats. They include stand-alone commercial security software by companies such as McAfee and Symantec; built-in browser components, such as the safe browsing feature of Chrome; and third-party browser extensions, such as the Firefox browser add-on by Samuel and Zhang [2009] for preventing cross-site request forgery.

The community and browser extension provided by Web of Trust (mywot.com) is most closely related to our work. Its users score websites on four aspects of vendor reliability, trustworthiness, privacy, and child safety. They can also provide free-text comments, but the system does not process these comments automatically.
The key difference between SmartNotes and Web of Trust is that it applies machine learning to the information collected from various sources, and integrates it with information obtained by automated processing of the free-text comments.

\section{Web Scam Detection}

The web scam is a type of cybersecurity threat, in which a website makes false or intentionally misleading claims. Examples are fraudulent promises to help find work at home or cure various diseases, usually with the purpose to trick its visitors into providing their sensitive personal or financial information, such as credit card numbers. Such fraudulent sites often look legitimate and use effective deception techniques, including scientific references, patented claims, and testimonials.

The problem of detecting web scams is closely related to spam email detection, which is a well-researched area with high detection accuracy [Cormack, 2007a]. Some spam emails contain links to scam websites [Anderson et al., 2007] and we used this fact to create one of our datasets described later. The successful techniques for spam detection are based on analysis of email contents.

Another related problem is web spam [Gyöngyi and GarciaMolina, 2005], where attackers trick search engines and receives an undeservedly high rank for their web pages, with the purpose of attracting more visitors. There are two types of approaches for detection of web spam, called content-based and link-based [Becchetti et al., 2008]. Currently, the contentbased approaches tend to be more effective. The underlying techniques are almost similar to those used for detecting email spam [Cormack, 2007b; Cormack, 2010].

The current approaches to spam detection have limited success in detection of scam website because it is relatively easy for the scammers to adapt their content. We improve the detection results by integrating automatically collected heterogeneous information about websites.

\section{DESIGN OF SMARTNOTES}

The developed system help users to share their experiences related to web threats. Users can rate websites, post comment, and ask or answer questions about websites. We describe the main features of SmartNotes from the users' point of view and then give more details of the underlying components. We have implemented the initial system as a Chrome browser extension. We plan to implement SmartNotes for other browsers as part of the future work. 


\section{A. User Interface}

To install SmartNotes, the user clicks the related link on the distribution website at cyberpsa.com. After installing the extension, Chrome displays a button for invoking it to the right of the address bar (Figure 1).

When the user wants to make a comment or ask a question regarding the currently displayed website, she clicks the SmartNotes button, which brings up the main window (Figure 1). The user can select the rating (positive, neutral, or negative), name the related bookmark, and add a free-text comment. When she clicks the Save button, the system sends these data to the server.

The user can share her notes and questions with others, using the options in Figure 2. The recipient can follow a link in the email to post a response. If the selected recipient is Friends or Everyone, the system automatically selects one or more individuals to ask the question. The user can see the number of unanswered questions and unread responses in the main window as shown in Figure 1.

By default, the notes are for the currently open web page, but the user can also post notes for the entire domain containing the current page. For instance, when she is looking at a specific product on Amazon, she may post notes about that product page or about the entire amazon.com service. The user can specify whether her notes are private, visible to her friends, or publicly available for everyone. When others visit a website with public notes, they can see the number of notes and click to read them. Users can also browse a database of all websites with that have notes and search within this database. In addition, the system allows importing the links to the commented pages into the browser bookmarks.

The user can invoke the automatic scam detection through the Analyze option, as shown in Figure 3. When the user clicks it, the system contacts the Host Analyzer web service, which collects the necessary information about the current website and then calculates the probability that the current domain name is a scam, called ScamScore.

\section{B. System Components}

The system consists of three main components as shown in Figure 4, which communicate through HTTP requests.

1) Browser extension: This component is written in JavaScript and uses Chrome extension API to interact with the browser. We used jQuery JavaScript library (jquery. com) to create a graphical user interface. All the processing is done in remote web services to simplify our updating mechanism.

2) SmartNotes web service: This component is written in C\#.NET and communicates with a SQL Server database. It exposes methods for reading and writing notes, as well all other features available in the user interface, such as account creation, login, and creating and retrieving the user contacts.

3) Host Analyzer web service: This component is also written in C\#.NET and stores all the data in the file system. It includes the machine learning algorithms, described in the next section, that compute ScamScore, as shown in Figure 4.

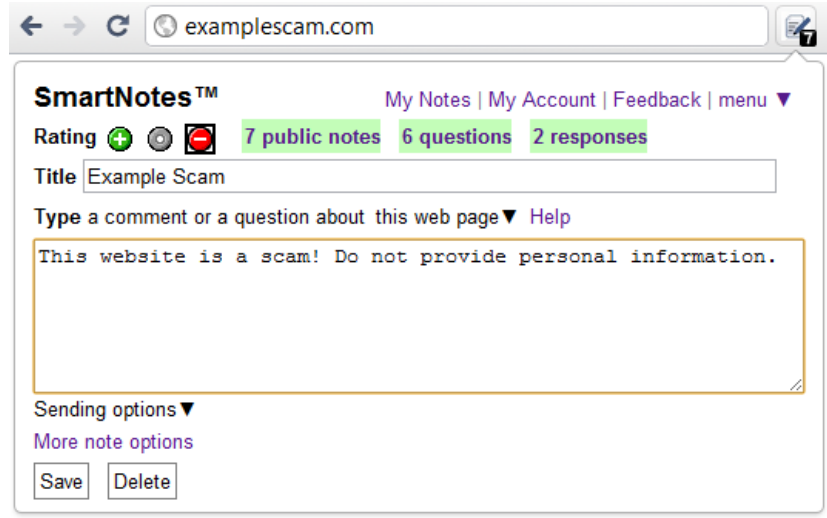

Figure 1. Screenshot of the SmartNotes browser extension.

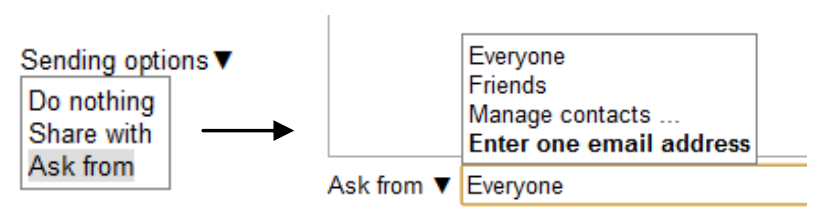

Figure 2. Sending options, which allow sharing or asking questions from others users regarding the current website.

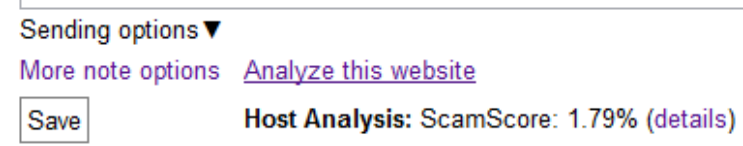

Figure 3. Link to invoke Host Analyzer web service.

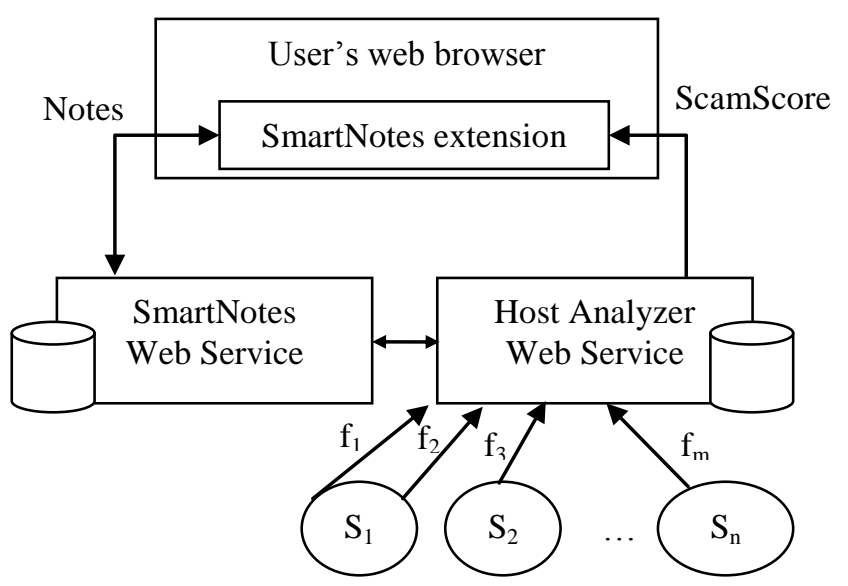

Figure 4. The SmartNotes architecture. The arrows show communications over HTTP. Host Analyzer web service calculates ScamScore based on $m$ features from $n$ sources. It also uses the notes from the SmartNotes web service. 
For data collection, it has a set of HTML wrappers that extract specific information from web pages. To improve this data collection, we use multithreading and caching. This web server has higher bandwidth, processing power, and storage capacity requirements than the web server for the SmartNotes web service.

\section{Web Scam Detection TeChNique}

We have created a web service for collecting information about websites. It includes a collection of HTML wrappers, which are small programs that extract useful parts of the web pages. We refer to pieces of the collected information as features. For example, traffic_rank is an integer feature obtained from alexa.com. The current version of the system collects 43 features from 11 sources (Table 1).

We need a dataset of websites labeled as scam or not scam to apply our machine learning technique. We have used the following approaches to construct this dataset.

1. Scam queries: Certain search queries are especially likely to return a large number of scam websites. We issued the following three Google queries and recorded the top 500 results for each query: "cancer treatments", "work at home", and "mortgage loans". After removing the documents, videos and duplicates, we randomly picked 100 domain names for each query and submitted them to the Amazon Mechanical Turk for manual labeling. For each link, we collected three human opinions. The instruction was for the labeler to review the website and decide whether it is scam or not scam and also provide a level of confidence (highly confident, somewhat confident or not confident). The final dataset contains websites that all three labelers agreed on the label with high confidence.

2. Web of Trust: To collect a set of popular links, we crawled the 200 most recent threads on the "Comments on websites" forum of the Web of Trust (mywot.com). We extracted all domain names mentioned in these discussion threads, which resulted in 159 unique domain names. To add diversity in this dataset, we added the websites ranked 1-200, 1000$1100,10000-10100$, and $15000-15100$ on alexa.com. We eliminated any websites with less than 5 comments on the Web of Trust. We then sorted the remaining 456 websites by the human provided score. We used top 150 websites as negative and bottom 150 websites as positive instances.

3. Spam emails: We obtained a dataset of 1551 spam emails from a corporate email system, detected with high confidence by McAfee AntiSpam in November 2010. We extracted 11825 links from those emails. Since spam emails sometimes provide links to legitimate high-ranked websites to trick the spam filters, we eliminated all links that are listed in the top one million websites on alexa.com. We then identified the links that occurred at least ten times and used them as scam examples.

4. hpHosts: To test the effectiveness of our approach on new threats, we took the 100 most recent reported website on the blacklist at hosts-file.net.

\begin{tabular}{|c|c|c|}
\hline \multicolumn{3}{|l|}{ Source (URL) } \\
\hline Feature Name & \multicolumn{2}{|c|}{ Definition } \\
\hline \multicolumn{3}{|l|}{ Google (google.com) } \\
\hline \multicolumn{2}{|c|}{ search_result_count } & $\begin{array}{l}\text { Number of search result when } \\
\text { query is the domain name. }\end{array}$ \\
\hline \multicolumn{3}{|l|}{ Alexa (alexa.com) } \\
\hline reviews count & \multicolumn{2}{|c|}{ Number of user comments. } \\
\hline rating & \multicolumn{2}{|c|}{ Overal website rating. } \\
\hline traffic rank & \multicolumn{2}{|c|}{ Worldwide traffic rank. } \\
\hline us traffic rank & \multicolumn{2}{|c|}{ US traffic rank. } \\
\hline \multirow{2}{*}{$\begin{array}{l}\text { sites linking in } \\
\text { IP Info (ipinfodb.com) }\end{array}$} & \multicolumn{2}{|c|}{ Number of website linking to this website. } \\
\hline & & \\
\hline $\begin{array}{l}\text { latitude, } \\
\text { longitude }\end{array}$ & \multicolumn{2}{|c|}{$\begin{array}{l}\text { Coordinate for the physical server } \\
\text { location. }\end{array}$} \\
\hline country code & \multicolumn{2}{|c|}{ Country code of the server location. } \\
\hline \multicolumn{3}{|c|}{\begin{tabular}{l|l} 
ip_count & Number of ip addresses
\end{tabular}} \\
\hline \multicolumn{3}{|l|}{ Wh } \\
\hline \multicolumn{2}{|c|}{ country code } & $\mathrm{Cou}$ \\
\hline \multirow{2}{*}{\multicolumn{2}{|c|}{\begin{tabular}{l|l}
$\begin{array}{l}\text { created_days, } \\
\text { updated_days, } \\
\text { expires days }\end{array}$ & $\begin{array}{l}\text { Number } \\
\text { update or } \\
\text { name. }\end{array}$ \\
\multicolumn{2}{l}{ Wikipedia (wikipedia.org) }
\end{tabular}}} & \\
\hline & & \\
\hline \multicolumn{2}{|l|}{$\begin{array}{l}\text { years_in_business, } \\
\text { company_revenue, } \\
\text { employees }\end{array}$} & $\begin{array}{l}\text { Years since the company's } \\
\text { establish date, yearly revenue } \\
\text { and number of employees. }\end{array}$ \\
\hline \multicolumn{3}{|c|}{ Google Finance (google.com/finance) } \\
\hline market cap & \multicolumn{2}{|c|}{ Company's market capitalization. } \\
\hline febrowsing ( & 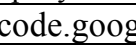 & ng) \\
\hline Whether & & \\
\hline mpete.c & & \\
\hline unique monthly & tors & visit. \\
\hline monthly visit & & isits. \\
\hline c rank & & \\
\hline truste.cor & & \\
\hline \begin{tabular}{l|l} 
member & Whether th \\
\end{tabular} & & \\
\hline mywot.com) & & \\
\hline rank & hased 0 & ore. \\
\hline popularity & & re oy wo \\
\hline $\begin{array}{l}\text { trustworthiness, } \\
\text { trustworthiness_con } \\
\text { vendor_reliability, } \\
\text { vendor_reliability_ } \\
\text { privacy, privacy_co } \\
\text { child_safety, } \\
\text { child_safety_conf }\end{array}$ & , & $\begin{array}{l}\text { Score given by the } \\
\text { community to each of these } \\
\text { aspects and the confidence } \\
\text { (i.e., number of votes } \\
\text { received). }\end{array}$ \\
\hline $\begin{array}{l}\text { total_positive_cc } \\
\text { total_negative_cc }\end{array}$ & $\begin{array}{l}\text { nents, } \\
\text { nents }\end{array}$ & $\begin{array}{l}\text { Total number of comments } \\
\text { left by the member for this } \\
\text { websites. }\end{array}$ \\
\hline $\mathrm{MIC}$ & & \\
\hline $\begin{array}{l}\text { site_is_good, } \\
\text { site_is_spam, ma } \\
\text { pop_ups, scam, } \\
\text { bad_shopping_exp } \\
\text { browser exploits }\end{array}$ & 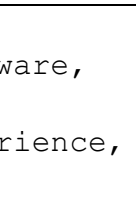 & $\begin{array}{l}\text { The number of community } \\
\text { votes for each of categories. }\end{array}$ \\
\hline total comments & & Sum of above numbers. \\
\hline
\end{tabular}

Table 1 The website features used for web scams detection. 
5. Top websites: This set is the top 100 websites according to the ranking on alexa.com. We used them as examples of non-scam.

We have applied the Host Analyzer to create a feature vector for each of the websites in the resulting dataset and then applied an L1-regularlized logistic regression algorithm. ScamScore in Figure 3 is the output probability of the website being scam shown as percentage. We performed evaluation on our method using 10-fold cross validation and measured precision (ratio of correctly predicted positives to total of predicted positives), recall (ratio of correctly predicted positives to total of positives in dataset), F1 (harmonic mean of precision and recall) and AUC (the area under the ROC curve). The results are summarized in Table $\mathbf{1}$.

\begin{tabular}{lcccc} 
Dataset Name & Precision & Recall & F1 & AUC \\
\hline Scam Queries & 0.9833 & 0.9667 & 0.9749 & 0.9667 \\
Web of Trust & 0.9923 & 0.9923 & 0.9923 & 0.9990 \\
Spam Email & 1.0000 & 0.9627 & 0.9809 & - \\
hpHosts & 1.0000 & 0.9200 & 0.9583 & - \\
Top Websites & 1.0000 & 0.9600 & 0.9795 & - \\
\hline Combined & 0.9795 & 0.9813 & 0.9803 & 0.9858 \\
\hline
\end{tabular}

Table 1 Web scam detection evaluation results.

\begin{tabular}{lr} 
Item & Count \\
\hline Comments & 170 \\
Max per user & 46 \\
Users & 98 \\
Currently active & 66 \\
Have comments & 18 \\
Requests (indicates activity) & 214466 \\
Max per user & 51025 \\
Contacts & 86
\end{tabular}

Table 2 SmartNotes usage statistics.

\section{Concluding Remarks}

We have described a system that implements a novel solution to detection of web security threats. It is available for download at cyberpsa.com. We have released the initial version in September 2010 and the latest version in December 2010. We summarize the current usage statistic in Table 2. The future work will include integration the current spam-detection mechanism with the analysis of information extracted from the free-text comments. We also plan to package the system as an add-on for several other browsers, including Internet Explorer and Firefox.

\section{REFERENCES}

[Anderson et al., 2007] Anderson D. S., Fleizach, C., Savage, S., and Voelker, G. M., Spamscatter: characterizing internet scam hosting infrastructure. In Proceedings of 16th USENIX Security Symposium on USENIX Security Symposium (SS'07). 2007.

[Barth et al., 2008] Barth A., Jackson, C., and Mitchell, J. C., Robust defenses for cross-site request forgery. In Proceedings of the 15th ACM conference on Computer and communications security (CCS '08), pages 75-88. 2008.

[Becchetti et al., 2008] Becchetti, L., Castillo, C., Donato, D., Baeza-Yates R., and Leonardi, S., Link analysis for Web spam detection. ACM Transactions on the Web, 2(1):1-42. 2008.

[Cormack, 2007a] Cormack, G. V., TREC 2007 Spam Track Overview. In Proceedings of TREC 2007: The Sixteenth Text Retrieval Conference. 2007.

[Cormack, 2007b] Cormack, G. V., Content-based Web spam detection. In Proceedings of the 3rd International Workshop on Adversarial Information Retrieval on the Web (AIRWeb). 2007.

[Cormack et al., 2010] Cormack, G. V., Smucker, M. D., and Clarke, C. L. A., Efficient and Effective Spam Filtering and Re-ranking for Large Web Datasets. Unpublished Manuscript. 2010.

[Cova et al., 2010] Cova, M., Kruegel, C, and Vigna, G., Detection and analysis of drive-by-download attacks and malicious JavaScript code. In Proceedings of the Nineteenth International Conference on World Wide Web, pages 281290. 2010.

[Barth and Garcia-Molina, 2005] Gyöngyi, Z., and GarciaMolina, H., Web Spam Taxonomy. First International Workshop on Adversarial Information Retrieval on the Web (at the 14th International World Wide Web Conference). 2005.

[Kumaraguru et al., 2009] Kumaraguru, P., Cranshaw, J., Acquisti, A., Cranor, L., Hong, J., Blair, M. A., and Pham, T., School of phish: A real-world evaluation of anti-phishing training. In Proceedings of the Fifth Symposium on Usable Privacy and Security, pages 1-12. 2009.

[Samuel and Zhang, 2009] Samuel, J. and Zhang, B., RequestPolicy: Increasing web browsing privacy through control of cross-site requests. In Proceedings of the Ninth Privacy Enhancing Technologies Symposium. 2009.

[Sharifi et al., 2010] Sharifi, M., Fink, E., and Carbonell, J. G., Learning of personalized security settings. In Proceedings of the IEEE International Conference on Systems, Man, and Cybernetics. 2010. 